\title{
Spectral graph analysis of the geometry of power flows in transmission networks
}

\author{
Nicolas Retière, Dinh Truc Ha, and Jean-Guy Caputo
}

\begin{abstract}
Power flows in transmission networks are driven by the structure of the network and the spatial distribution of generators and loads. Understanding the interplay between grid, generators and loads is crucial for efficient and robust planning and management of electrical networks. Using the spectral properties of the graph Laplacian, we show that we can express power flows on the basis of Laplacian eigenvectors and reveal the dominant modes for nodal voltages and branch powers. The bus voltages are dominated by low rank modes. The power in the lines depends on the nodal distribution of generators/loads, the line impedances and the gradient of the eigenvectors across the branches. The most loaded lines and their associated dominant mode are then identified. A modification of the bus powers is finally proposed to better share the load between the lines and hence to decrease the system vulnerability. Results provided for several IEEE transmission test systems prove the relevance of the approach and suggest practical guidelines to improve the operation of power systems.
\end{abstract}

Index Terms-Spectral graph theory, Laplacian, nodal domain, power system, power flow, vulnerability.

\section{INTRODUCTION}

$\mathbf{P}$ OWER grids cope with extreme stress due to the increasing demand, the financial pressure from electricity market, the growth of interconnections between regions or countries, the rise of distributed energy resources and the spread of digital technologies [?], [?], [?]. Hence, a single failure may lead to a major collapse and the disconnection of millions of customers. Since the 2000s, more than ten blackouts have impacted more than one billion people around the world [?]. They have not only cost millions of US dollars but have also affected other important infrastructure that deliver essential services such as communication, internet, transport, water and emergency services [?]. The analysis and evaluation of vulnerability of power systems have therefore become a key issue for power engineers and researchers [?]. To prevent major system security issue, it is required to reveal vulnerabilities in power grids. But, because of the system's increasing complexity, new methods are necessary to investigate their structural vulnerabilities [?]. These methods are required to localize and rank the elements less susceptible to absorb hazards, due to their high level of stress in normal operation. The factors and inputs potentially dangerous for

N. Retière and D.-T. Ha are with Université Grenoble Alpes, CNRS, G2elab, 38000 Grenoble, France e-mail: nicolas.retiere@univ-grenoblealpes.fr

J.-G. Caputo is with INSA de Rouen Normandie, Laboratoire de Mathématiques, 76801 Saint-Etienne du Rouvray, France

This work benefited from the support of the project FRACTAL GRID ANR15-CE05-007-01 of the French National Research Agency (ANR). structural vulnerabilities are also expected to be explained by these approaches.

Power flow calculation is a fundamental tool for the security analysis of power systems. It can be of use to predict security boundaries, to locate weakest elements, to assess loading of lines, transformers or generators, to verify that voltage values respect the autorized limits [?], Since power system$\mathrm{s}$ are networks composed of many interacting components, predicting, interpreting, understanding the vulnerabilities are difficult tasks that require many numerical simulations [?]. The coupling of power flow simulations with complex science has been of great benefit for the engineering community to analyze the results and figure out how power flows distribution and vulnerabilities are driven by the complex structure of electrical networks [?], [?]. A number of studies in the literature have already linked the graph topology of a network to its power flows [?]. Many are based on centrality: which nodes or links are most prominent for the flow of the electrical power in the grid. These geometric approaches evaluate the node degree, the betweenness, the clustering coefficient, to characterize a given topology [?], [?], [?], [?]. Unfortunately, in most cases, these approaches fail to predict with accuracy the power distribution in the network and in particular the most loaded lines. Actually, a central vertex for the geometry may not be central for the power flow. The absence in the centrality-based approaches of the distribution of generators and loads is the main reason of their failure to identify the weakest elements. Alternative approaches use spectral graph theory i.e. the study of the spectral properties of the characteristic matrices of the network. An application of spectral graph theory is clustering: the identification of sets of nodes that present similarities. This method is based on the computation of the eigenvector related to the second smallest eigenvalue of the Laplacian or adjacency matrix of the graph [?]. An extension of this procedure was applied to power systems where the network was partitioned into hierarchical clusters, using the k-th first eigenvectors of a graph matrix [?]. Graph partitioning is strongly connected to the dynamic coherency because it allows to identify groups of generators having similar dynamics. The fundamental work [?] draws theoretical links between dynamic coherency and the confinement of eigenvectors to some sets of nodes. Beyond clustering, spectral analysis of the graph Laplacian was applied to understand the links between the grid structure and its synchronization and stability properties [?], [?], [?], [?], [?]. The identification of the key nodes that drive the network's function can also be performed by spectral analysis of the adjacency matrix [?], [?]. Spectral graph theory was also applied to system security. For example, in [?], the 
nodal domains related to Laplacian eigenvectors provided the localization of potentially dangerous inputs for the system stability. To improve grid robustness, a lowering of the voltage drops was seeked in [?] by maximizing the second lowest eigenvalue of the Laplacian matrix. The second eigenvalue and its related eigenvector was also used to determine the critical edges of a transmission network [?]. Spectral approaches were finally used in some articles to compute the pseudo-inverse of the network's admittance matrix and thus solve the power flow equations. This allowed to easily compute the redistribution of currents when generators, loads or topology change [?], [?].

All the previous works describe the network's structure in terms of oscillatory modes. These modes are constituted by a couple of variables $\lambda$ and $\Psi$ where the eigenvalue $\lambda$ defines the wavelength and the eigenvector $\Psi$ gives the shape of the mode. Our first contribution is the comprehensive modeling of the power flows on the basis of such modes. Indeed, the DC approximation of power flow equations yields a linear system for the electrical variables [?], involving the graph Laplacian matrix (i.e. bus admittance matrix) and the vector of powers (generators and loads). The network topology information is contained in the Laplacian matrix while the power flow input/output is in the vector of powers. The Laplacian matrix is symmetric, positive so that its eigenvalues are real and one can choose a basis of orthonormal eigenvectors to write and solve the power flow equations. This was partially done in [?], [?] but we go one step further, developing the spectral expressions of the power flow equations without requiring the calculation of the pseudo-inverse of the Laplacian matrix. In addition, we show the importance of the eigenvalues and eigenvectors characterizing the network. Eigenvalues provide length-like scales: the low eigenvalues correspond to large scales and vice-versa. Eigenvectors give a geometrical global picture of the network at these different scales. Writing and solving the DC load flow in the Laplacian spectral basis allows to separate each wave-length and "hear" the network on each eigenmode of the graph Laplacian, much like a drum [?]. We hence provide a geometric picture of the drivers of the electrical variables. Our second main contribution is the determination of the modes that dominate the nodal voltages and branch powers. We also show that localized eigenvectors can cause a large power flow in some lines. We summarize the roles of modes on the branch powers by a transfer function connecting the power in the lines to the spectral distribution of nodal powers. This transfer function is higher at low wave numbers corresponding to energy transfers on large distances in the network. It can however exhibit some local peaks at high numbers too. Finally, we identify the most loaded lines whose removal is most susceptible to impact the system and propose a way to reduce their number. To show the usefulness of the approach we apply our approach to four IEEE transmission test cases.

The article is organized as follows. A nomenclature of the variables and parameters is provided in Section II. Basic results on spectral graph theory are presented in section III. Section IV shows the solution of the DC approximated power flow in the spectral domain of the Laplacian matrix. We obtain the voltage angles using the eigenvalues and eigenvectors of the graph Laplacian. These angles are then used to compute the power in the lines. Sections V details the spectral results for the nodal voltage angles. Section VI shows how the modes affect line power flows and Section VII provides a spectral view of the vulnerabilities of the networks. Conclusions are given in section VIII.

\section{NOMENCLATURE}

$\begin{array}{ll}d & \text { Graph diameter } \\ e_{i} & \text { i-th vector of the standard basis } \\ f & \text { Function defined on the nodes } \\ i, j & \text { Nodes indices } \\ k & \text { Eigenvalue or mode rank } \\ m & \text { Number of edges of the graph } \\ n & \text { Number of nodes of the graph } \\ o_{k} & \text { k-th coordinate of } \theta \text { in the eigenbasis } \\ p_{k} & \text { k-th coordinate of } P \text { in the eigenbasis } \\ r & \text { multiplicity of the eigenvalue } \\ A[n \times n] & \text { Adjacency matrix of the graph } \\ D[n \times n] & \text { Degree matrix of the graph } \\ E & \text { Set of edges of the graph } \\ L[n \times n] & \text { Laplacian matrix of the graph } \\ N_{k} & \text { k-th nodal domain } \\ P & \text { Nodal power vector } \\ P_{i}[n \times 1] & \text { Active power of node } i \\ P_{L}[m \times 1] & \text { Branch power flow vector } \\ V & \text { Set of nodes of the graph } \\ Y_{L}[m \times m] & \text { Line admittance matrix } \\ \lambda_{k} & \text { k-th eigenvalue of the Laplacian matrix } \\ \Lambda[n \times n] & \text { Pseudo-inverse of the eigenvalue matrix } \\ \omega & \text { Edges weight } \\ \nabla[m \times n] & \text { Gradient over the graph edges } \\ \Psi_{k}[n \times 1] & \text { Eigenvector related to } \lambda_{k} \\ \Psi_{k}(i) & \text { i-th component of } \Psi_{k} \\ \theta[n \times 1] & \text { Nodal voltage angle vector } \\ & \end{array}$

\section{BASIC RESULTS ON SPECTRAL GRAPH THEORY}

\section{A. Graph Laplacian}

A graph $G$ is defined as $G=(V, E, \omega)$ where $V$ is the vertex set of size $n, E$ is the edge set of size $m$ along with the functions $\omega: E \rightarrow \mathbb{R}^{+}$for the weights. The weighted adjacency $A$ and degree matrices $D$ of the graph are given by:

$$
\begin{aligned}
& A(i, j)= \begin{cases}\omega_{i j} & \text { if } i, j \in E \\
0 & \text { otherwise. }\end{cases} \\
& D(i, j)= \begin{cases}\sum_{k=1}^{n} A(i, k) & \text { if } j=i, \\
0 & \text { otherwise. }\end{cases}
\end{aligned}
$$

The Laplacian matrix is defined by $L=D-A$.

Given a function $f$ defined on the nodes, the Laplacian can also be defined by the quadratic form [?]:

$$
f^{T} L f=\sum_{i, j \in E} \omega_{i j}(f(i)-f(j))^{2}
$$




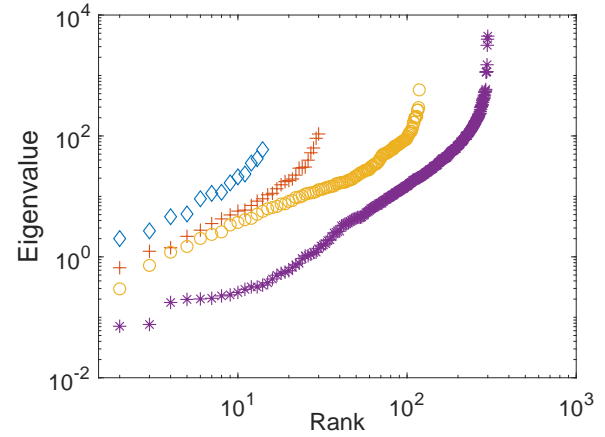

Fig. 1: Sorted eigenvalues

14-bus $(\diamond)$ - 30-bus (+) - 118-bus (o) - 300-bus (*)

\section{B. Eigenvalues and eigenvectors}

$\Psi$ is an eigenvector associated to eigenvalue $\lambda$ of $L$, then:

$$
L \Psi=\lambda \Psi
$$

Since the edge weights are all positive, the Laplacian matrix is a symmetric, positive-semidefinite matrix. Its eigenvalues are thus real and positive and they can be sorted out such that:

$$
\lambda_{1}=0 \leq \lambda_{2} \leq \ldots \leq \lambda_{n}
$$

The first eigenvalue is 0 because $L$ is singular; there is only one because we assume a connected graph. The smaller eigenvalues are related to the global connectivity of the network whereas the larger ones quantify the local behavior [?]. The eigenvectors associated to the different eigenvalues can be chosen to be orthogonal and form an orthonormal basis of $\mathbb{R}^{n}$. Using the quadratic form (??) of the Laplacian, it is easy to see that the eigenvector $\Psi_{1}$ associated to $\lambda_{1}=0$ has equal entries. Then since any eigenvector $\Psi_{k}(k>1)$ is orthogonal to $\Psi_{1}$, it verifies:

$$
\sum_{i=1}^{n} \Psi_{k}(i)=0
$$

Note that $\Psi_{k}(i)$ corresponds to the node $i$ of the network.

\section{Nodal domains}

A nodal domain $N_{k}$ associated with $\Psi_{k}$ is defined as a maximal connected subgraph of nodes with value of same sign [?]:

$$
i, j \in N_{k} \Leftrightarrow \Psi_{k}(i) \Psi_{k}(j) \geq 0
$$

The nodal domain is positive (resp. negative) if the value is positive (resp. negative). It is said to be strong if the product of eigenvector entries is strictly positive. In the case of weak domains, the value of some nodes of the domain may thus be zero. Many works are related to the number of domains. In a pioneer work, Fiedler gave first results about the nodal domains related to the second eigenvalue of nonnegative symmetric matrices [?]. In [?], Davies et al. provided generalized results for any eigenvector associated to eigenvalue of a simple (no self-loop) and undirected graph $\lambda_{k}$. They show that there are at most $k$ weak nodal domains and $k-r$ strong domains, $r$ being the multiplicity of the eigenvalue. One of the most recent result was demonstrated by Urschel [?]. For any eigenvalue, there exists an eigenvector that can be decomposed in at most $k$ nodal domains. Basically, these results mean that the network may be decomposed in two domains for the second eigenvalue and at most $n$ domains for the largest eigenvalue. We will see in the next section that nodal domains plays a major role in the distribution of power flows.

\section{SPECTRAL SOlution OF DC POWER FlOW}

\section{A. DC power flow}

In steady-state operation, any AC transmission network made of $n$ nodes and $m$ branches can be described by a set of $n$ algebraic equations. They link active and reactive powers, voltage magnitude and angle. They are called power flow equations [?]. DC approximation is a well known technique to simplify power flow equations of meshed transmission systems [?]. Under this approximation, power flow equations are reduced to a linear matrix equation:

$$
L \theta=P
$$

where $P$ is the vector whose components are the powers at each bus. $\theta$ is a vector giving bus voltage angles and $L$ is the DC bus admittance matrix. By construction, it is equal to the Laplacian matrix associated with the underlying weighted graph of the network. Edge weights are the susceptances of the lines.

\section{B. Spectral solving}

Instead of solving the DC power flow in the standard basis of the vertex space of the graph, the orthonormal basis consituted by the eigenvectors associated the eigenvalues of Laplacian matrix is used. The standard basis is defined as a set of vectors $\left(e_{1}, e_{2}, \ldots e_{n}\right)$ where $e_{i}$ is a column vector of size $n$ associated to vertex $i$. Its components are all equal to zero except the i-th entry which is equal to $1 . P$ is expressed in terms of standard basis elements by:

$$
P=\sum_{i=1}^{n} P_{i} e_{i}
$$

where $P_{i}$ is the active power injected on node $i$.

Projecting $P$ onto the orthonormal basis $\Psi_{1}, \Psi_{2}, \ldots \Psi_{n}$, we get:

$$
P=\sum_{k=1}^{n} p_{k} \Psi_{k}
$$

where $p_{k}$ is the projection of $P$ on $\Psi_{k}$.

Developing line by line this expression, we obtain:

$$
P_{i}=\sum_{k=1}^{n} p_{k} \Psi_{k}(i)
$$

The same projection is used for voltage angles such that:

$$
\theta=\sum_{k=1}^{n} o_{k} \Psi_{k}
$$




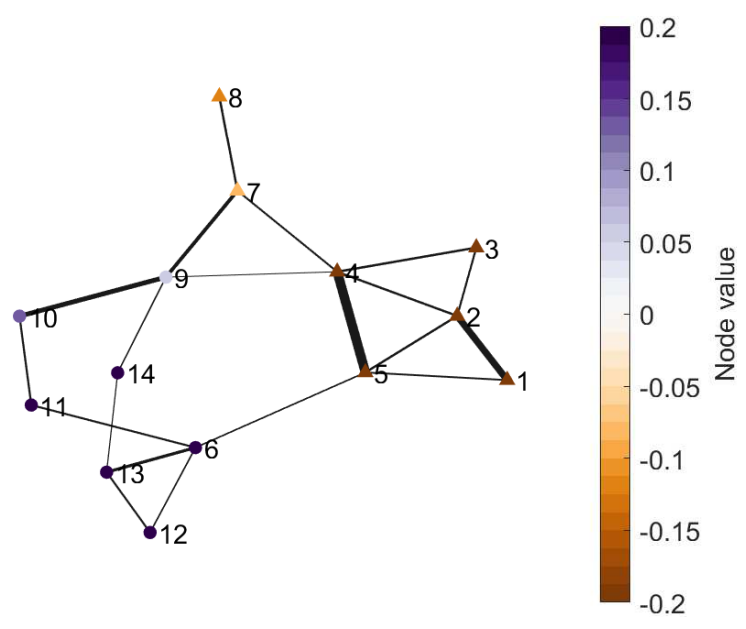

(a) 14-bus

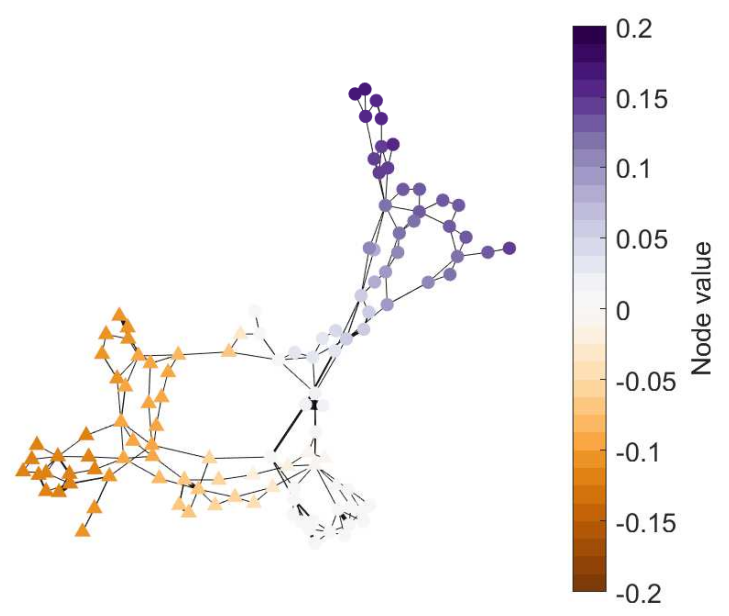

(c) 118-bus

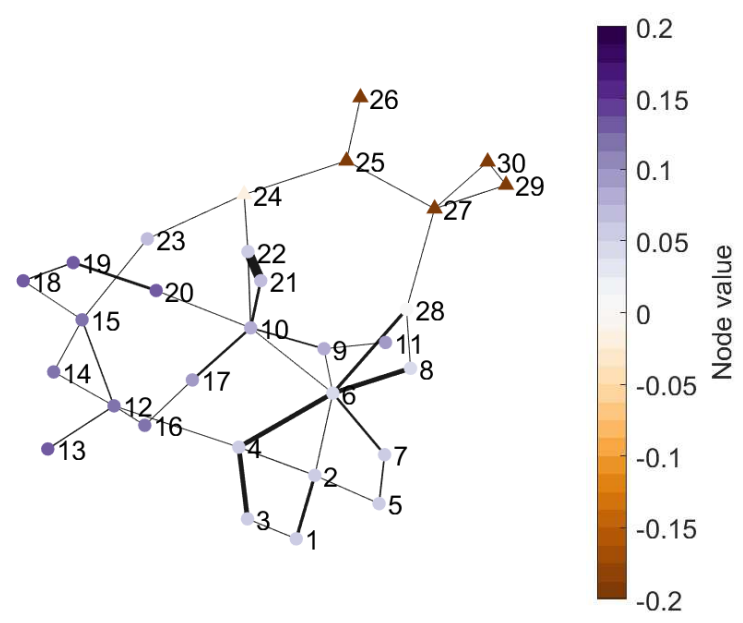

(b) 30-bus

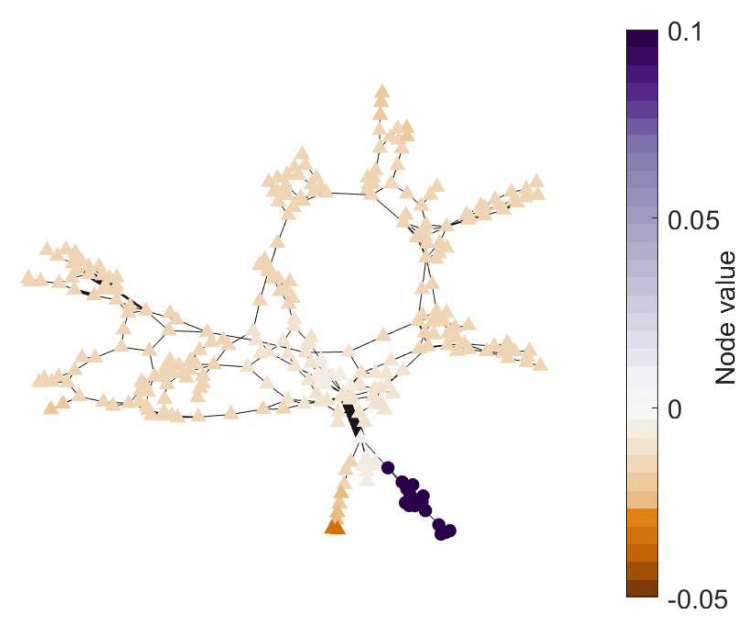

(d) 300-bus

Fig. 2: $\lambda_{2}$-nodal domain - $\triangle$ : negative nodes - $\circ$ : positive nodes.

Because power systems are balanced in steady-state operation:

$$
\sum_{i=1}^{n} P_{i}=0
$$

It comes:

$$
\sum_{i=1}^{n} \sum_{k=1}^{n} p_{k} \Psi_{k}(i)=0
$$

This can be rewritten, permutating the sums:

$$
p_{1} \sum_{i=1}^{n} \Psi_{1}(i)+\sum_{k=2}^{n} p_{k} \sum_{i=1}^{n} \Psi_{k}(i)=0
$$

The second term of the right hand side is zero because of formula (??). Since all the entries $\Psi_{1}(i)$ are equal and non zero, we get $p_{1}=0$.

Now, we come back to the DC power flow equation (??) and express the vectors onto the eigenvector basis. This yields:

$$
\sum_{k=1}^{n} p_{k} \Psi_{k}=L \sum_{k=1}^{n} o_{k} \Psi_{k}
$$

Due to eigenvector definition, it can be expressed as:

$$
\sum_{k=1}^{n} p_{k} \Psi_{k}=\sum_{k=1}^{n} o_{k} \lambda_{k} \Psi_{k}
$$

Then, since the eigenvectors are orthogonal, we get:

$$
p_{k}=\lambda_{k} o_{k}
$$

The computation of phase angle coefficients is straightforward. For all $k>1, \lambda_{k} \neq 0$ and:

$$
o_{k}=\frac{p_{k}}{\lambda_{k}}
$$

The component $o_{1}$ is undetermined because the system is balanced $\left(p_{1}=0\right)$ and the first eigenvalue is zero. Actually, the term $o_{1}$ is the reference angle of the network; we choose it equal to zero for simplicity.

To summarize, we have reduced the solution of the full $[n \times$ $n]$ system described by (??), to the evaluation of $n$ independent spectral responses (??). This fundamental simplification results from the orthogonality of the eigenvectors $\Psi_{k}$. 


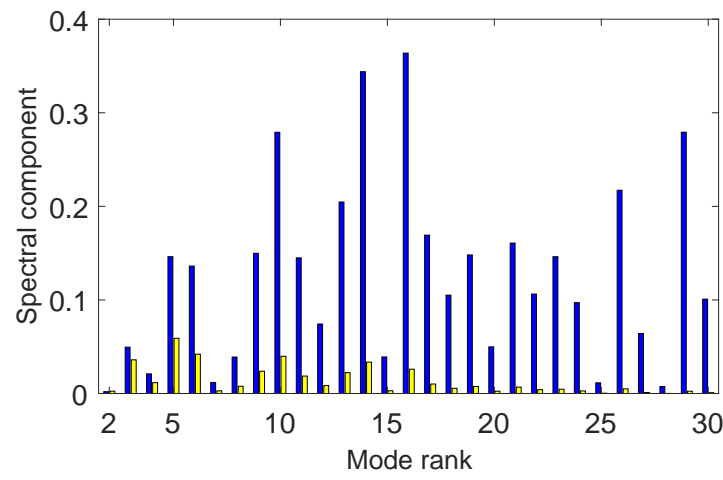

Fig. 3: 30-bus - Power flow spectral results $\left|o_{k}\right|$ (yellow) - $\left|p_{k}\right|$ (blue)

\section{Calculation of the flow on lines}

The power flow on the transmission lines of the grid can be expressed as:

$$
P_{L}=Y_{L} \nabla \theta
$$

where $P_{L}$ is a vector made of branch power flows, $Y_{L}$ is a diagonal matrix representing the line admittances and $\nabla$ is the gradient over the edges of the graph. Given a function $f$ on the vertices, it is defined by:

$$
\nabla f(e)=f(i)-f(j), \quad e=(i, j) \in E
$$

It is worth noting that the gradient can be also defined as the transpose of the link-node incidence matrix of the graph [?].

In terms of spectral variables, the power on line $i$ is expressed by:

$$
P_{L}(i)=Y_{L}(i, i) \sum_{k=1}^{n} \frac{p_{k}}{\lambda_{k}} \nabla \Psi_{k}(i)
$$

Apart from the eigenvalue $\lambda_{k}$, the line admittance $Y_{L}(i, i)$ and the power distribution on modes $p_{k}$, the gradient of eigenvector components over the edges is a key variable for power flow on lines. It is related to the structure of nodal domains.

Inside a domain, the edges connect vertices that have with the same sign. Then the gradients over the edges are expected to be smaller than for edges at the border between two adjacent domains. To justify this, recall that a nodal domains is the maximal subgraph that gather all the vertices with values of same sign. Then, two adjacent domains are of opposite sign and at their border, the gradient over the edges could be large.

\section{Spectral AnAlysis of Voltage ANGLES}

Four test systems are studied in this section. They are all meshed and their number of nodes vary from 14 to 300 . Network data and reference results are provided by Matpower free Matlab package [?].

\section{A. Eigenvalues and eigenvectors of the test cases}

The eigenvalues of the four test cases are arranged in the increasing order and plotted in the log-log diagram of figure ??. Eigenvalues $\lambda_{1}$ that are equal to zero are not plotted.

For homogeneous graphs (no weigths on the edges), the spectral distribution is bounded from above. We have [?]:

$$
\lambda_{n} \leq \max (D(i, i)+D(j, j)), \quad(i, j) \in E
$$

where $D(i, i)$ is the degree of node $i$.

The algebraic connectivity $\lambda_{2}$ verifies [?]:

$$
\frac{4}{n d} \leq \lambda_{2}
$$

where $d$ is the diameter of the graph, meaning the number of edges in the shortest path between the most distant vertices. For such homogeneous systems, as the number of vertices is increased, the spectral distribution extends towards zero. It is bounded from above if the degrees of the nodes do not vary much. Electrical networks are more complex because of weights. We see from figure ?? that the spectral distributions extend towards zero as the number of buses increases. Its upper bound increases slowly with $n$, perhaps due to the weights.

The nodal domain decomposition for the algebraic connectivity of the four test cases are plotted in figure ??. The domains are indicated by the color and the shape of the nodes markers. The size of the edges is also proportional to the weight (admittance value). As predicted by the theory, we see two nodal domains for each network. However, their structure differs significantly due to the geometry and connectivity of the network. On one hand, for the 118-bus case, the admittance values are similar for all the lines and the network is separated into two balanced domains. On the other hand, for the 300bus case, the positive domain is confined to a fork which is connected to the rest of network by a line of very high admittance.

The graph clustering provided by the second smallest eigenvalue is a first approach to study system vulnerability. It provides the minimum number of branches that should be removed to cut the graph in two independent parts [?], [?]

\section{B. Power flow spectral solution}

Spectral calculation of power flows is performed by using equations (??), (??) and (??). The results have been compared with DC power flow computation performed by Matpower. Results are very consistent and confirm that this spectral approach is a way to calculate power flows under DC assumptions. The drawback that lies behind its apparent simplicity is the computation cost of eigenvalues and eigenvectors that can be expensive for very large systems. And the spectral solution is only valid for very simple loads and generators models. Its main advantage is to provide a deep insight on the links between graph geometry and transmitted powers. It complements the methods of solving AC and DC power flows, see the recent advances [?], [?].) 


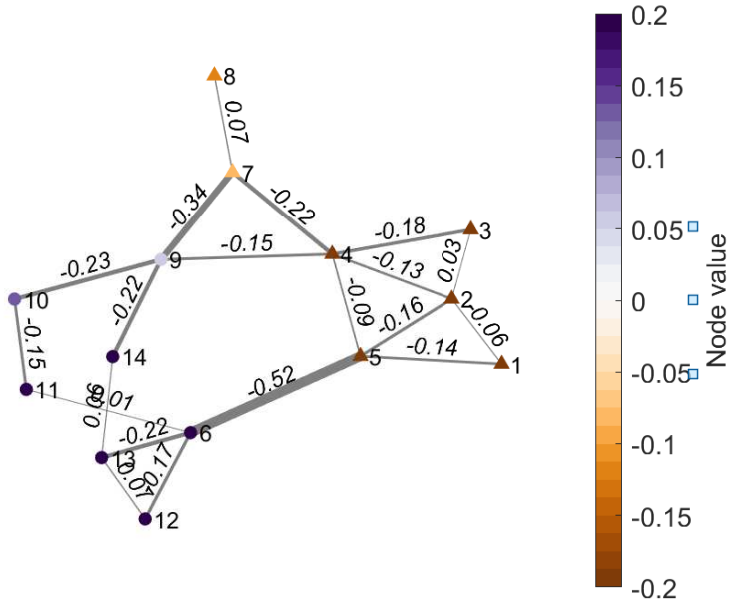

Fig. 4: 14-bus - Power on lines if nodal power is distributed on mode 2 only $\left(p_{k}=0\right.$ for $k \neq 2$ ) - Line width proportional to carried power

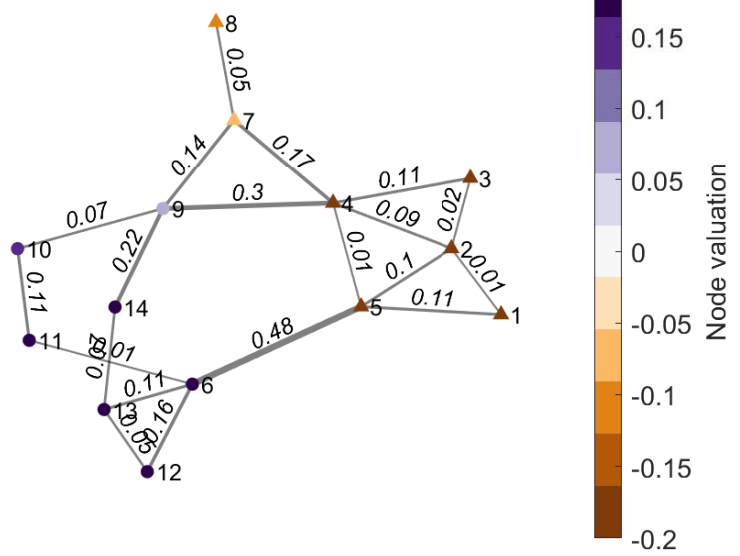

Fig. 5: 14-bus - Gradient of eigenvector components over the edges on mode 2 - Line width proportional to gradient

\section{Dominant modes}

$p_{k}$ and $o_{k}$ are linked by the inverse of the eigenvalue $\lambda_{k}$ (see equation (??)) and eigenvalues increase rapidly with the rank as shown in figure ??. So, even if nodal powers are distributed over a large range of modes, the angles are dominated by the lower modes. This is shown for the case 30 in figure ??. The absolute values of the spectral components $p_{k}$ and $o_{k}$ are plotted along the mode rank $k$. The power is distributed over all modes $k$ but modes below 15 dominate the voltages which appear to be controlled by power transfers at longer distances. We will see below that the higher modes are determinant for the loading of the branches.

\section{SPECTRAL ANALYSis OF THE POWERS ON LINES}

Our main purpose is now to understand how the graph Laplacian modes drive the powers on the lines. Equation (??) shows that the powers on the lines depend on the eigenvalues $\lambda_{k}$, the gradient of eigenvectors over edges $\nabla \Psi_{k}$ (and so the structure of nodal domains), the line admittances values $Y_{L}$

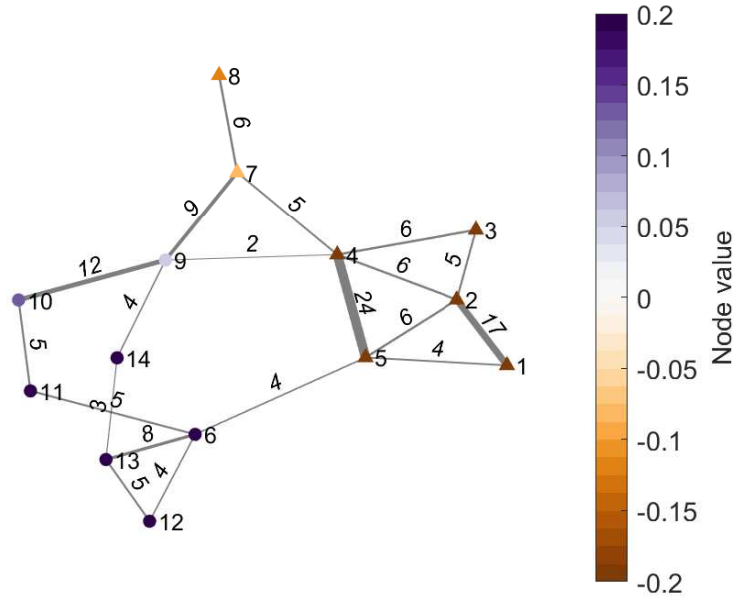

Fig. 6: 14-bus - Network line admittances - Line width proportional to admittance $\left(\Omega^{-1}\right)$

and the location of sources and loads which is parametrized by $p_{k}$. They are the components of the projection of the nodal power vector $P$ on the eigenbasis as shown in equation (??).

Two cases are now examined. In this section, the single mode excitation, meaning that the total load power is only on one mode, is studied. In the next section, the nominal excitation, where the power vector is defined in each Matpower test case, will be detailed.

\section{A. Single mode excitation}

We first study the power on lines when the total power of the network is distributed on a single mode $k_{0}$. This means that all the components $p_{k}$ are equal to 0 except $p_{k_{0}}$. The nodal power vector is thus expressed by:

$$
P=p_{k_{0}} \Psi_{k_{0}}
$$

A node $i$ is generating power if $\Psi_{k_{0}}(i)$ is positive and is a load node if $\Psi_{k_{0}}(i)$ is negative.

The power on line $i$ is then given by:

$$
P_{L}(i)=Y_{L}(i, i) \frac{p_{k_{0}}}{\lambda_{k_{0}}} \nabla \Psi_{k_{0}}(i)
$$

The case $k_{0}=2$ is shown in figure ?? for the 14-bus case. The values of powers flowing through the lines are indicated near the edges. They are normalized by the total load power fed into the network. The line width of the edges is also proportionnal to these values. The values of the power flows result from the combination of the eigenvector gradient and the admittance values as shown in figures ?? and ??. The gradient values drive the power flow on lines 5-6. A high power flow is also reached on lines 7-9 because of its relatively low impedance and high gradient. It is worth mentionning that the vertices 4 and 5 are very well connected (very high admittance value) but belong to the same domain (very low eigenvector gradient). The power flow between these two nodes is thus relatively low.

The same analysis can be carried out for the other modes. A summarized view of the results is presented in figure ?? for the 


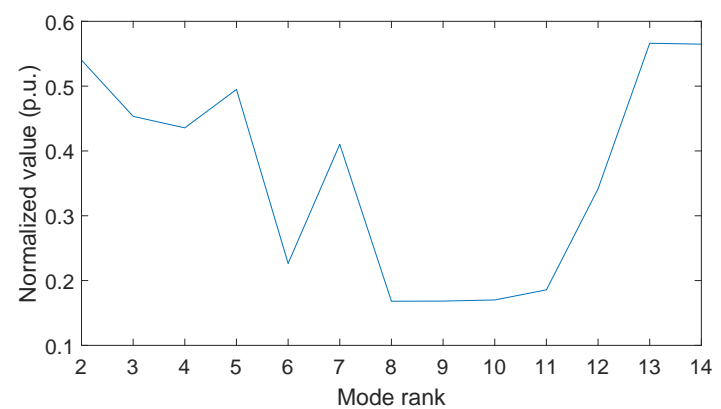

(a) 14-bus

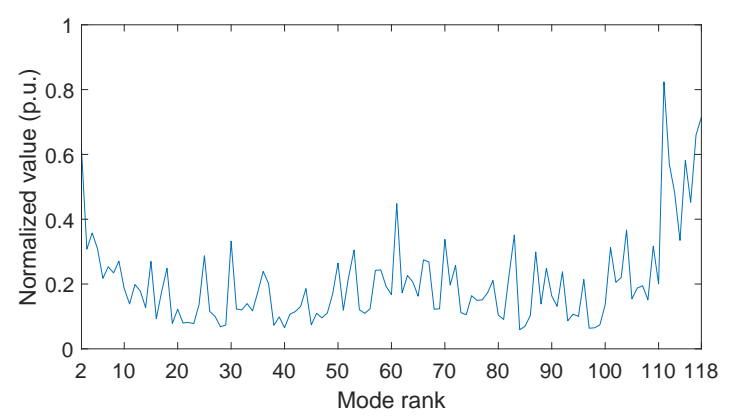

(c) 118-bus

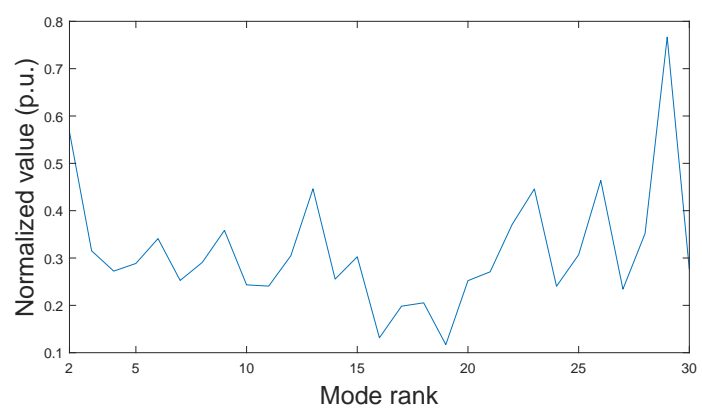

(b) 30-bus

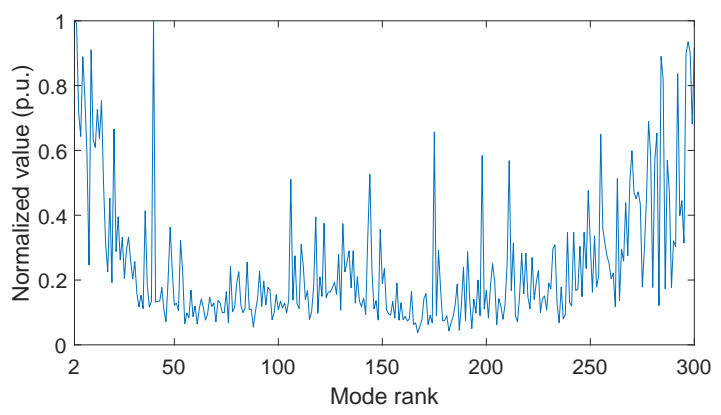

(d) 300-bus

Fig. 7: Maximal power on lines.

four test cases. The maximal value reached by powers on lines in every mode is plotted. The values are again normalized by the total load power in order to compare the four test networks. A general trend can be observed. The highest values tend to be reached in the lower and higher modes. According to the theory, for lower eigenvalues, the network is decomposed into very few domains. The loads are concentrated in the negative nodal domains and the generators are located in the positive domains. The interconnection lines between these domains may be very loaded due to this uneven location of power elements. For higher eigenvalues, the network may be fragmented into a lot of small-size domains (whose number is at most equal to the rank of the eigenvalue). Some lines might be locally sollicitated to transfer a high amount of power between two neighbouring domains.

An illustration of this phenomenon is given for the 30-bus case. In figure ??, the power flow distribution is shown for $\lambda_{29}$. The i-th component of the eigenvector related to this eigenvalue is affected to the $\mathrm{i}$-th node. Most of the eigenvector entries are zero except for two neighbouring vertices. At the node 22 with positive value, all the generation is connected whereas the total load is connected at the node 21. Of course, the line between these two vertices is very loaded. The power flow and eigenvector structure is more distributed for the eigenvalue $\lambda_{30}$ as shown in figure ??. This is the reason why the maximum power flow is not as high as in the 29-th mode.

A summarized view of the eigenvectors entries is given for the 118- and 300-bus test cases. For the 118-bus case and lower eigenvalues, the eigenvectors are uniformly distributed over the vertices (see the right-hand side of figure ??). But for higher ranks, the eigenvectors appear to be very localized (high values on few nodes). The structure of the 300-bus eigenvectors is not similar (cf. figure ??). Lower modes are surprisingly localized. This result has already been observed for the nodal domain of $\lambda_{2}$ on figure ??. Its negative domain is restricted to a very few number of vertices. In any case, the "excitation" of localized modes shall be avoided to not cause a local excess of power flows on some lines.

\section{B. The network as a transfer function}

Coming back to equation (??), the line power flow can be expressed under a matrix form such as:

$$
P_{L}=Y_{L} \nabla \Psi \Lambda^{-1} p
$$

Where $p=\left[p_{1}, p_{2} \ldots, p_{n}\right]^{T}$ and $\Lambda^{-1}$ is a diagonal matrix with diagonal entries equal to the inverse of the non-zero eigenvalues and zero for the first eigenvalue.

The network is therefore described by a transfer function given by the matrix $Y_{L} \nabla \Psi \Lambda^{-1}$. This matrix expresses the link between the input vector $p$ (the spectral distribution of generators and loads) and the output response (the power on lines $\left.P_{L}\right)$. Each entry of the transfer function describes the contribution of the mode $k$ on the line $i$. They are plotted in figure ??. They exhibit high peaks and deep valleys for lower rank modes and are more flattened with some localized peaks or valleys for higher ranks. For lower ranks, the transfer function of a transmission network is large to facilitate the transfer of power over the system. For higher ranks, at local scales, the transfer function is close to zero. This means 


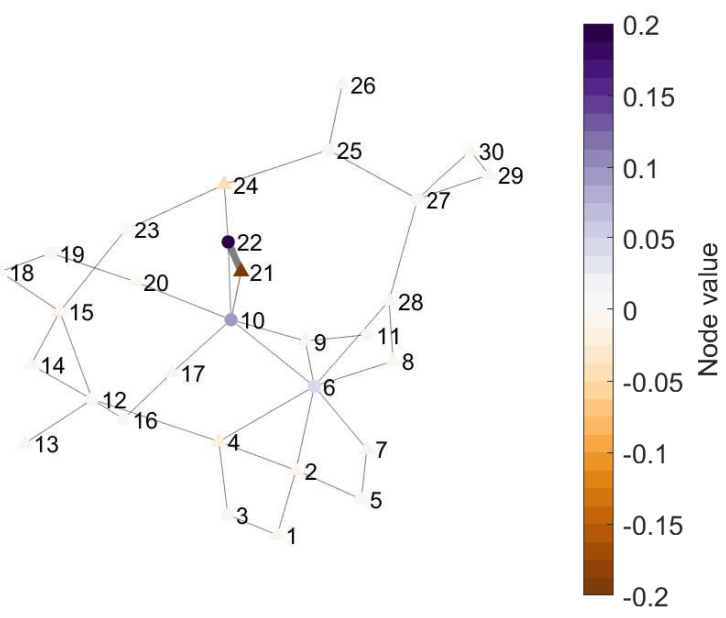

(a) Mode 29

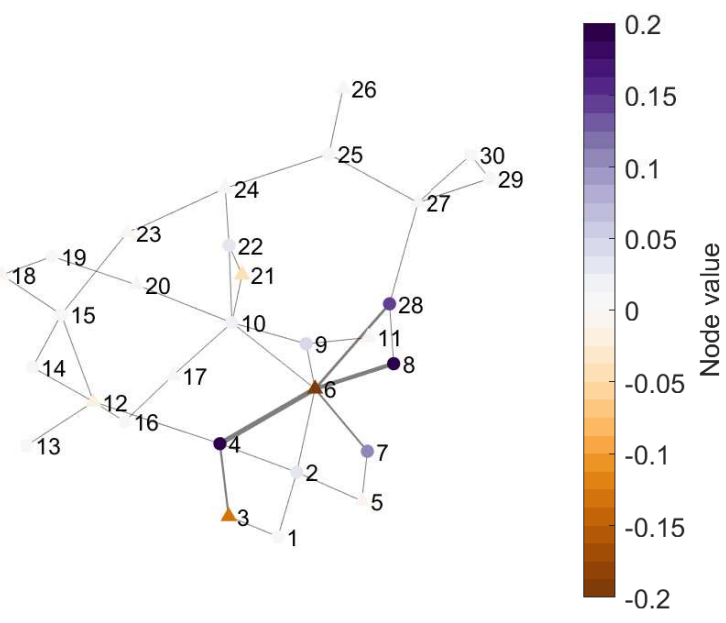

(b) Mode 30

Fig. 8: 30-bus - Line power distribution - Line width proportional to carried power.

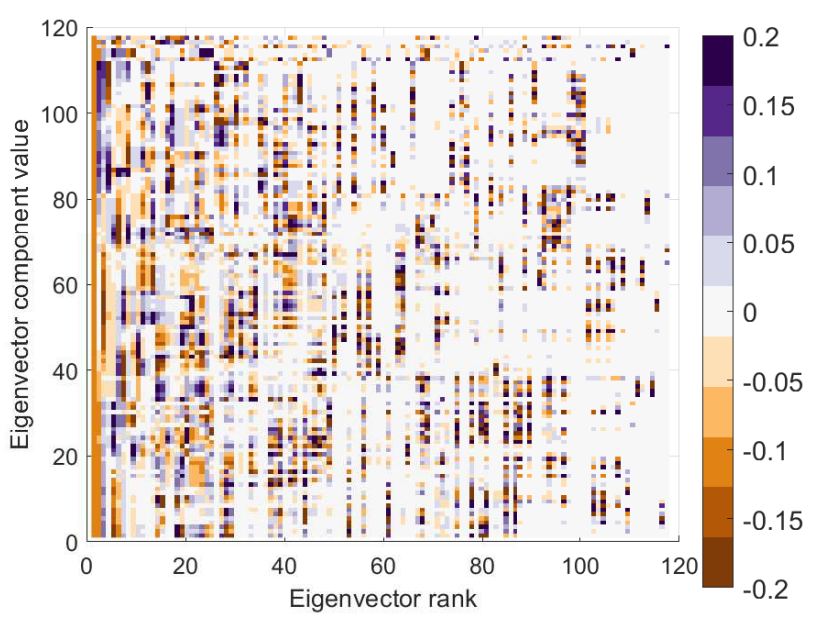

(a) 118-bus

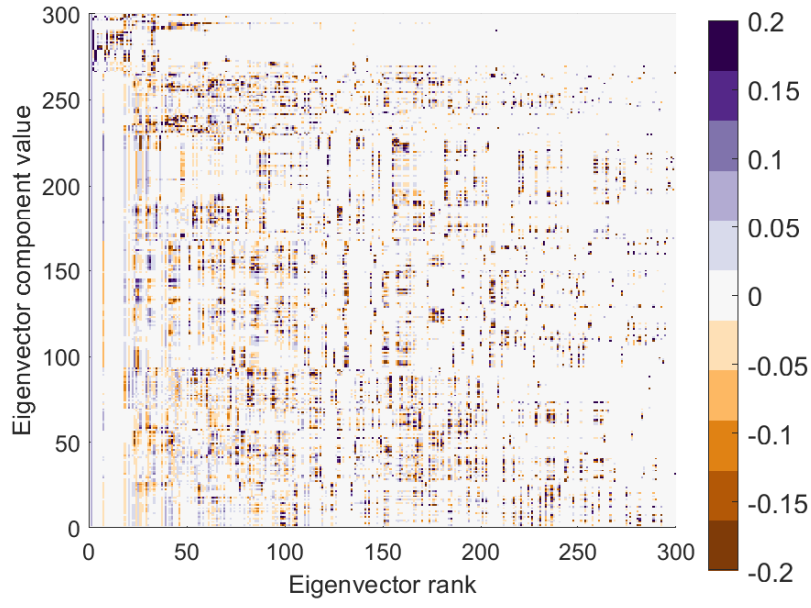

(b) 300-bus

Fig. 9: Eigenvector components.

that generally small amounts of power are transferred locally between nodes. But the excitation of the localized peaks and valleys shall be avoided to limit the loads on lines.

\section{SPECTRAL ANALYSIS OF VULNERABILITIES}

\section{A. Determination of the most loaded lines}

We now investigate the spectral features of the four nominal test cases and assess how and why some lines are more loaded than others. Nominal means that we use the nodal distribution of generators and loads as defined in the Matpower's test cases. The spectra $p=\left(p_{1}, p_{2}, \ldots p_{n}\right)$ of these nominal distributions are shown in figure ??. They are the input of the network's transfer function shown in figure ??. Notice that these inputs are more concentrated on the higher than the lower modes.

The absolute values of the resulting line powers are plotted in figure ??. They are normalized by the total load value. Of course, the lines are not evenly loaded. However, note the larger the network, the less loaded are the lines. Indeed, larger meshed transmission networks offer more paths for the power flow, therefore increasing the transmission capacity and limiting the risks of line congestions.

In the nominal cases, all the modes are excited and interplay in the power line calculation as expressed by equations (??) or (??). For example, the most loaded transmission element of the 30-bus nominal test case is the line 22 as shown in figure ??. Its spectral decomposition is given in figure ??. The most dominant mode is associated with $\lambda_{10}$. This results from the combination of the level of excitation of the 10-th mode (figure ??), the gradient value across the line 22 between the nodes 12 and 13 (figure ??) and the admittance of the line (its value is closed to the mean value of the line admittances of the test case). None of these values are very high but their combination give way to an overloading of line 22 when compared to the other lines.

The previous detailed analysis can be carried out for every test cases. The five most loaded lines and their associated dominant mode are given in table ??. These lines are the 


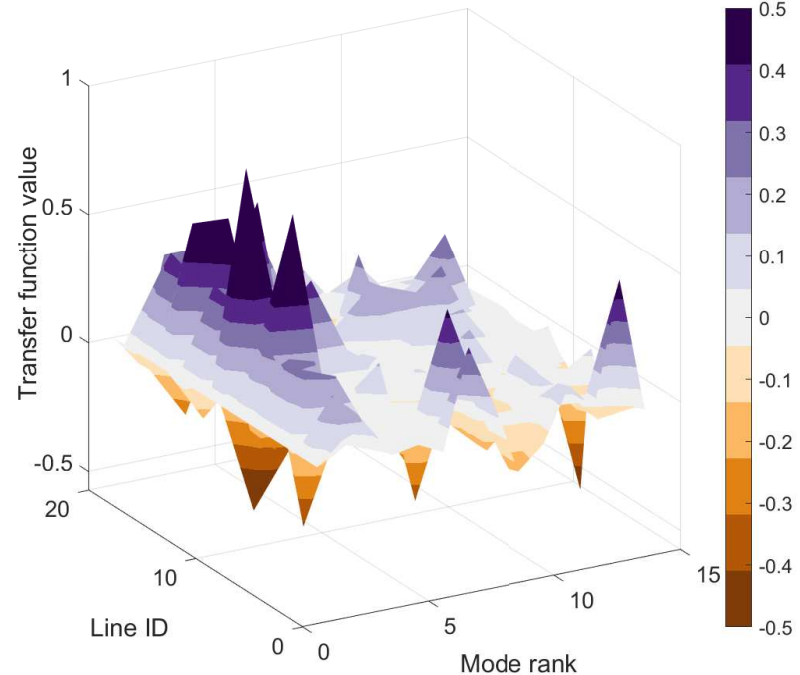

(a) 14-bus

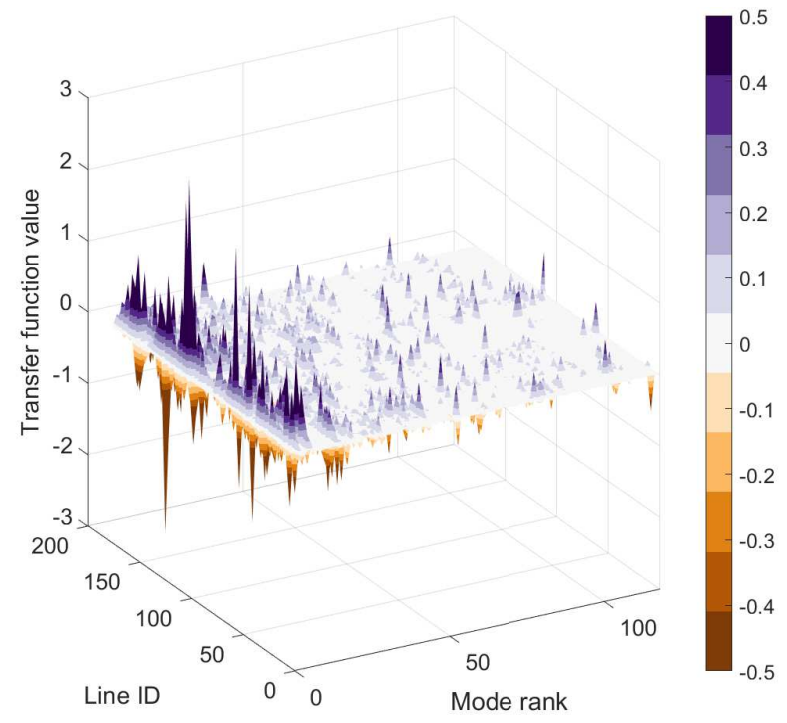

(c) 118-bus

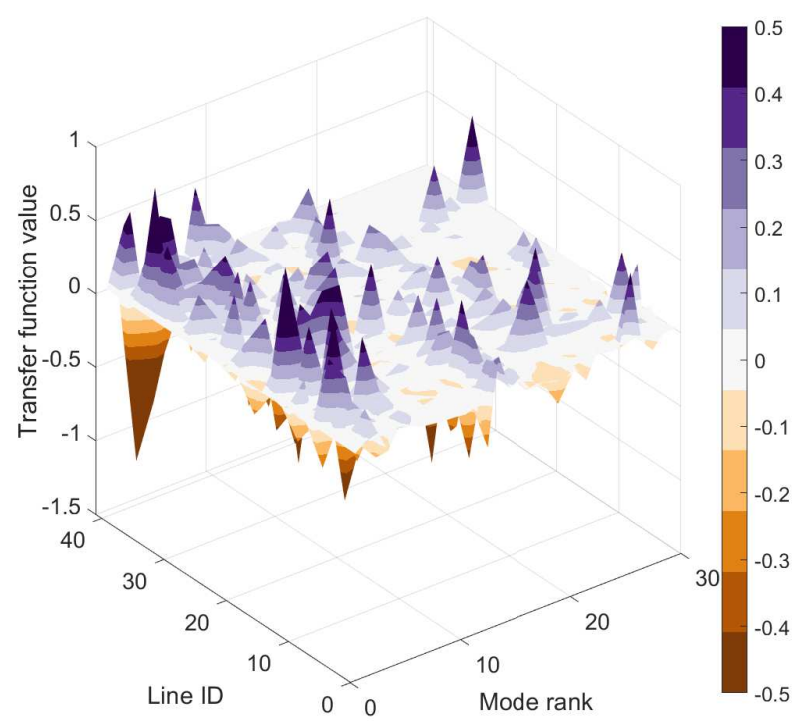

(b) 30-bus



(d) 300-bus

Fig. 10: Network transfer function - x-axis: $k$; y-axis: $i$; z-axis: $Y_{L}(i, i) \frac{1}{\lambda_{k}} \nabla \Psi_{k}(i)$.

least susceptible to absorb hazards due to their high level of stress in normal operation. The results clearly show that they are dominated by high rank modes, i.e. controlled by power transfers at shorter distances. These modes may also control more than one overloaded line. For a given graph structure, the choice of the spectral distribution of generators and loads parametrized by $p_{k}$ is therefore a crucial variable. It should be adjusted to better share the loads between lines and increase the system robustness.

\section{B. Reduction of the vulnerabilities}

An example of reduction of vulnerability is given for the 300-bus test case. As previously depicted, the spectral distribution of nodal powers in the nominal case produces very stressed lines. We have then decided to choose another input vector $p$ in order to offer a more uniform sharing between
TABLE I: Most loaded lines (sorted by descending order) and associated dominant mode

\begin{tabular}{|c|c||c|c||c|c||c|c|}
\hline \multicolumn{2}{|c||}{ 300-bus } & \multicolumn{2}{c||}{ 118-bus } & \multicolumn{2}{c|}{ 30-bus } & \multicolumn{2}{c|}{ 14-bus } \\
\hline Line & Mode & Line & Mode & Line & Mode & Line & Mode \\
\hline 216 & 166 & 13 & 15 & 22 & 10 & 1 & 7 \\
\hline 12 & 218 & 15 & 15 & 22 & 14 & 2 & 7 \\
\hline 296 & 173 & 9 & 18 & 22 & 14 & 3 & 7 \\
\hline 8 & 7 & 147 & 100 & 32 & 29 & 7 & 7 \\
\hline 221 & 218 & 81 & 71 & 5 & 14 & 4 & 7 \\
\hline
\end{tabular}

modes and thus avoid over-excitation of localized modes. The histograms of both nominal and new input vectors are shown in figure ??. This clearly indicates that the modified vector is more homogeneously distributed over the modes. This yields a better distribution of the powers on lines as shown in table ??. The mean value of the lines loading is lower than in the 


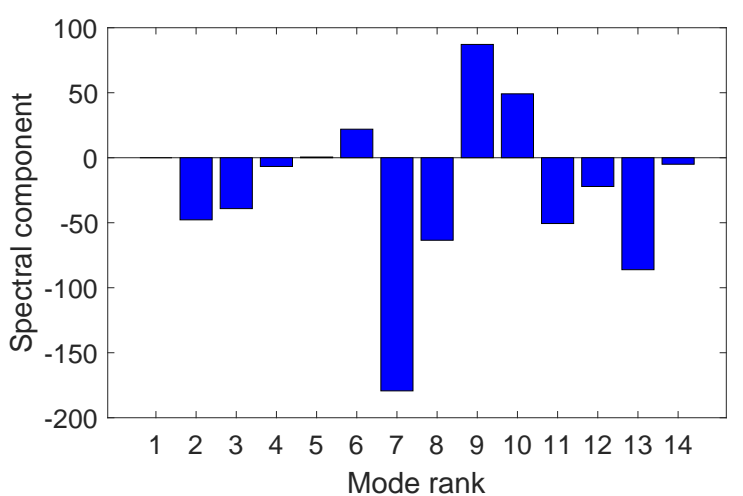

(a) 14-bus

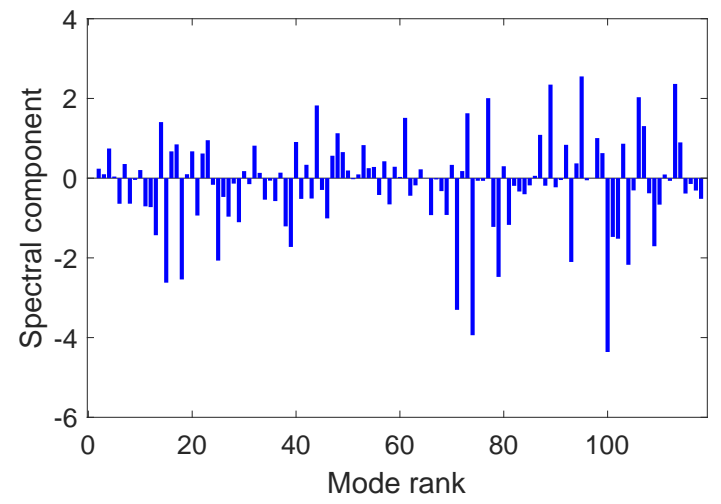

(c) 118-bus

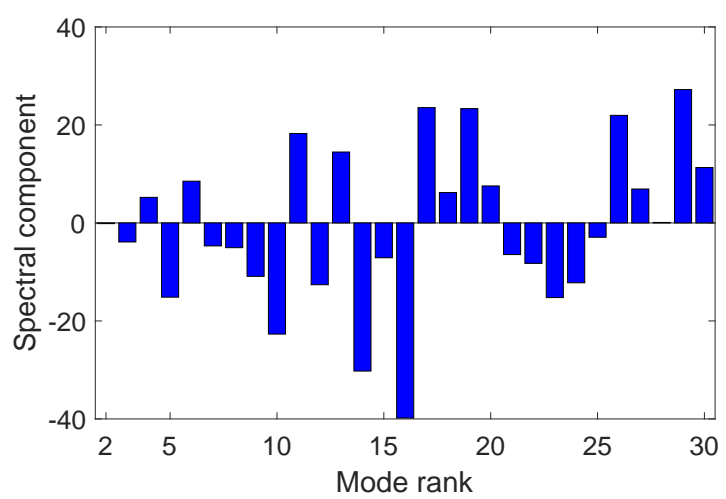

(b) 30-bus



(d) 300-bus

Fig. 11: Spectra $p=\left(p_{1}, p_{2}, \ldots p_{n}\right)$ of nodal powers for the nominal cases.

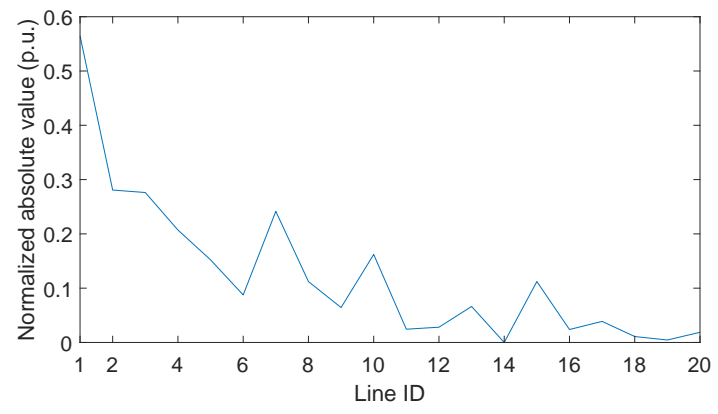

(a) 14-bus

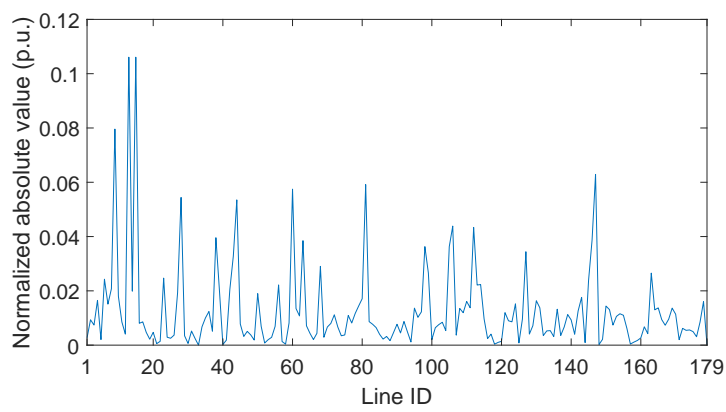

(c) 118-bus

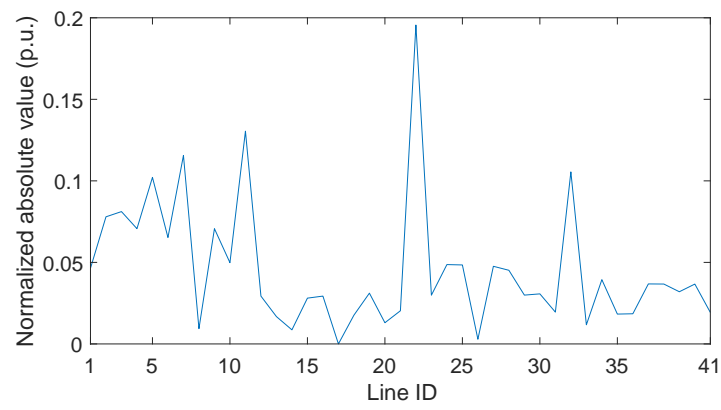

(b) 30-bus



(d) 300-bus

Fig. 12: Power on lines for the nominal cases. 


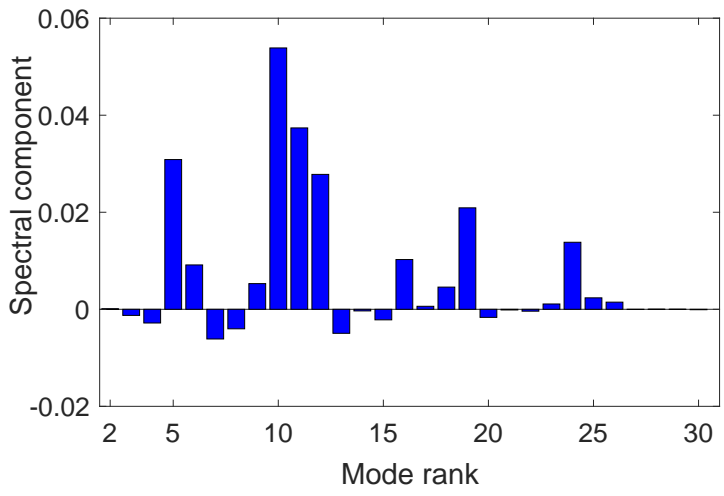

Fig. 13: 30-bus - Spectra of power on line 22

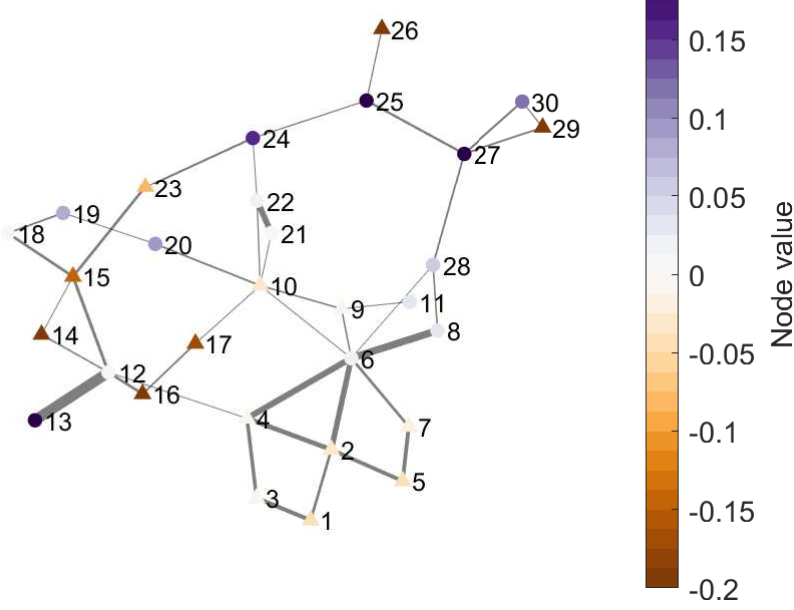

Fig. 14: 30-bus - Nodal domains for $\lambda_{10}$ - Line width proportional to carried power

TABLE II: Statistics on the power on lines (values in MW)

\begin{tabular}{|l||c||c||c|}
\hline & Mean & Standard deviation & Maximum \\
\hline Nominal case & 135 & 187 & 1292 \\
\hline Modified case & 84 & 34 & 173 \\
\hline
\end{tabular}

nominal case and the standard deviation is reduced meaning a more homogeneous sharing. Finally, the maximal load has been divided by more than 6 showing that the over-excitation of unwanted localized modes has been avoided.

\section{CONCLUSION}

We introduced a spectral approach to decompose and solve the DC power flow equations on the basis of the Laplacian eigenmodes whose characteristic length is given by the eigenvalues and geometrical shape is determined by the eigenvectors. This provides a geometric picture of the drivers of the electrical variables.

We find that bus voltages are mainly dominated by large scale modes corresponding to the low ranking eigenvalues. Line flows are affected by the nodal domains associated with the signed structure of the components of the eigenvectors. Another important parameter is the series admittance of the lines, for example low impedance lines may magnify some
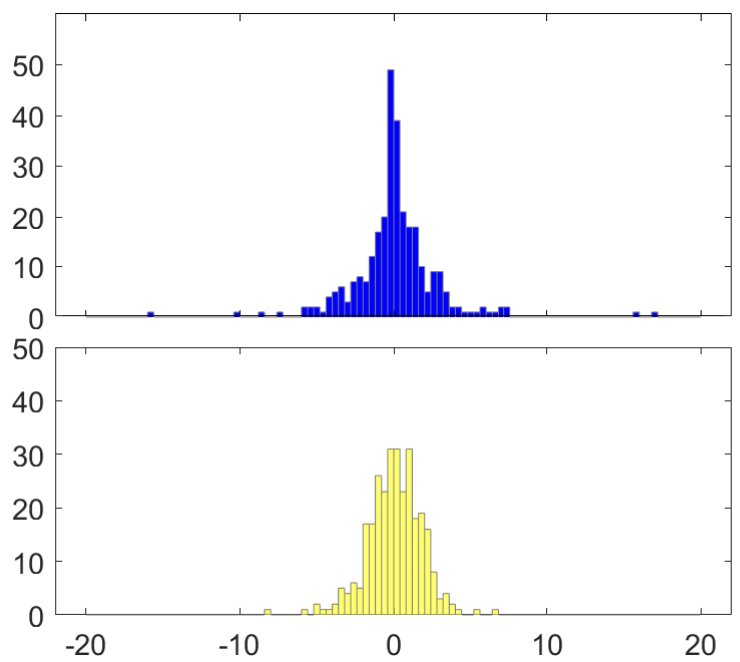

Fig. 15: 300-bus - Histograms of nominal (blue) and modified (yellow) input vectors $p$

power transfer as shown in equation (??). Unfortunately most known theoretical results in spectral graph theory assume equal admittances. To extend these results to unequal values is a challenging problem for graph theorists.

Finally, we introduce a transfer function connecting the power in the lines $P_{L}$ to the generator/load distribution $p$. This transfer function is large for low wave numbers corresponding to energy transfers on large length scales of the network. High numbers exhibit some localized peaks.

A practical consequence is that electrical engineers designing power systems should avoid high ranking eigenmodes that are very localized because these can cause overloaded lines (table ??). An example of the modification of the power distribution was suggested to better share the loads between the lines and therefore to reduce the system vulnerability.

The challenge open now for power engineers is to select the optimal power input taking into account the spectral structure of the network and the operational constraints.

\section{REFERENCES}

[1] S. Massoud Amin and B. F. Wollenberg, "Toward a smart grid: power delivery for the 21st century," in IEEE Power and Energy Magazine, vol. 3, no. 5, pp. 34-41, Sept.-Oct. 2005.

[2] E. Kuznetsova, K. Culver, and E. Zio, "Complexity and vulnerability of Smartgrid systems," in Proc. European Safety and Reliability Conference, Troyes, France, 2011, pp. 2474-2482.

[3] ETP Smart grids, "Strategic research agenda or europe's electricity networks of the future," 2013. [Online]. Available: URL http://www.smartgrids.eu/documents/sra2035.pdf, June 2013.

[4] M. Velay, M. Vinyals, Y. Besanger, and N. Retière, "An analysis of large scale transmission power blackouts from 2005 to 2016," in Proc. UPEC Conference, Glasgow, Scotland, UK, Sept. 4-7, 2018, .

[5] S. Küfeoğlu, "Economic impacts of electric power outages and evaluation of customer interruption costs," $\mathrm{PhD}$. Dissertation, Aalto University, Aalto, Finland, 2015

[6] L. Luo, B. Han, and M. Rosas-Casals, "Network Hierarchy Evolution and System Vulnerability in Power Grids", in IEEE Systems Journal, vol. 12, no. 3, pp. 2721-2728, Sept. 2018.

[7] E. Bompard, R. Napoli, and Fei Xue, "Analysis of structural vulnerabilities in power transmission grids,", in International Journal of Critical Infrastructure Protection, vol. 2, no. 1-2, pp. 5-12, 2009.

[8] P. Kundur, Power System Stability and Control, McGraw-Hill Professional, New-York, 1994. 
[9] S. Arianos, E. Bompard, A. Carbone, and F. Xue, "Power grid vulnerability: A complex network approach," inChaos, vol. 19, no. 013119, 2009.

[10] N. Rubido, "Energy Transmission and Synchronization in Complex Networks: Mathematical Principles," PhD. Dissertation, Aberdeen University, Aberdeen, Scotland, UK, 2014.

[11] E. Winsberg, "Computer Simulations in Science", in The Stanford Encyclopedia of Philosophy, Edward N. Zalta (ed.), Standford, Californa, USA: Metaphysics Research Lab, 2019.

[12] S. Boccaletti, V. Latora, Y. Moreno, M. Chavez, D. Hwang, "Complex networks: Structure and dynamics", inPhysics Reports, vol. 424, no. 4-5, pp. 175-308, 2006.

[13] P. Hines, S. Blumsack, E. C. Sanchez and C. Barrows, "The Topological and Electrical Structure of Power Grids," in Proc. 43rd Hawaii International Conference on System Sciences, Honolulu, HI, 2010, pp. 1-10.

[14] E. I. Bilis, W. Kröger and C. Nan, "Performance of Electric Power Systems Under Physical Malicious Attacks," in IEEE Systems Journal, vol. 7, no. 4, pp. 854-865, Dec. 2013.

[15] Y. Wang, J. Zhao, F. Zhang and B. Lei, "Study on structural vulnerabilities of power grids based on the electrical distance," in Proc. IEEE PES Innovative Smart Grid Technologies, Tianjin, China, 2012, pp. 1-5.

[16] T. Werho, V. Vittal, S. Kolluri and S. M. Wong, "Power System Connectivity Monitoring Using a Graph Theory Network Flow Algorithm," in IEEE Transactions on Power Systems, vol. 31, no. 6, pp. 4945-4952, Nov. 2016.

[17] S. E. Schaeffer, "Graph clustering," in Computer Science Review, vol. 1, no. 1, pp. 27-64, Aug. 2007.

[18] R. J. Sánchez-García et al., "Hierarchical Spectral Clustering of Power Grids," in IEEE Transactions on Power Systems, vol. 29, no. 5, pp. 22292237, Sept. 2014

[19] B. Ayazifar, "Graph spectra and modal dynamics of oscillatory networks," PhD. Dissertation, Massachusetts Institute of Technology, Cambridge, MA, 2002.

[20] T. R. Nudell and A. Chakrabortty, "A graph-theoretic algorithm for localization of forced harmonic oscillation inputs in power system networks," in emphProc. 2014 American Control Conference, Portland, OR, 2014, pp. 1334-1340.

[21] T. Ishizaki, A. Chakrabortty, and J. Imura, "Graph-Theoretic Analysis of Power Systems," in Proceedings of the IEEE, vol. 106, no. 5, pp. 931-952, May 2018.

[22] F. Dörfler, M. Chertkov, and F. Bullo, "Synchronization in complex oscillator networks and smart grids," Proceedings of the National Academy of Sciences, vol. 110, no. 6, pp. 2005-2010, 2013.

[23] A. Diwadkar and U. Vaidya, "Synchronization in large-scale nonlinear network systems with uncertain links," in Automatica, vol. 100, pp. 194199, Feb. 2019

[24] L. Guo, C. Zhao and S. H. Low, "Graph Laplacian Spectrum and Primary Frequency Regulation," in Proc. IEEE Conference on Decision and Control (CDC), Miami Beach, FL, 2018, pp. 158-165.

[25] V. Fioriti, M. Sforna, and G. D'Agostino, "Spectral analysis of a real power network," in International Journal of Critical Infrastructures, vol. 8, no. 4, pp. 354-367, 2012.

[26] J. Li, L. Dueñas-Osorio, C. Chen, B. Berryhill, and A. Yazdani, "Characterizing the topological and controllability features of U.S. power transmission networks," in Physica A: Statistical Mechanics and its Applications, vol. 453, pp. 84-98, Jul. 2016.

[27] A. Torres and G. Anders, "Spectral Graph Theory and Network Dependability," in Proc. Fourth International Conference on Dependability of Computer Systems, Brunow, 2009, pp. 356-363.

[28] E. Goddet, N. Retière, V. Stojanović, A. Dieudonné, J. Genoulaz, and J.M. Guichon, "Maximizing the algebraic connectivity of meshed electrical pathways used as current return network," in Mathematics and Computers in Simulation, vol. 158, 2019, pp. 18-31.

[29] V. Rosato, S. Bologna, and F. Tiriticco, "Topological properties of high-voltage electrical transmission networks," in Electric Power System Research, vol. 77, no. 2, pp. 99-105, 2007.

[30] Cetinay, H., F. A. Kuipers, and P. Van Mieghem, "A Topological Investigation of Power Flow", in IEEE Systems Journal, vol. 12, no. 3, pp. 2524-2532, 2018

[31] B. Stott, J. Jardim, and O. Alsac, "DC Power Flow Revisited", in IEEE Transactions on Power Systems, vol. 24, no. 3, pp. 1290-1300, Aug. 2009.

[32] M. Kac, "Can one hear the shape of a drum?," in American Mathematical Monthly, vol. 73, no.4, pp. 1-23, 1966.

[33] R. Merris, "Laplacian matrices of graphs: a survey," in Linear algebra and its applications, vol. 197, pp. 143-176, 1994.
[34] E. B. Davies, G.M.L. Gladwell, J. Leydold, and P. F. Stadler, "Discrete nodal domain theorems," in Linear Algebra and its Applications, vol. 336, no. 1-3, pp. 51-60, 2001.

[35] M. Fiedler, "A property of eigenvectors of nonnegative symmetric matrices and its application to graph theory," in Czechoslovak Mathematical Journal, vol. 25, no. 4, pp. 619-633, 1975.

[36] J. C. Urschel, "Nodal decompositions of graphs", in Linear Algebra and its Applications, vol. 539, pp. 60-71, 2018.

[37] J.-G. Caputo, A. Knippel, and E. Simo, Oscillations of networks: the role of soft nodes, in Journal of Physics A: Mathematical and Theoretical, vol. 46, no. 3, pp. 035101, Jan. 2013.

[38] R. D. Zimmerman, C. E. Murillo-Sánchez, and R. J. Thomas, "MATPOWER: Steady-State Operations, Planning and Analysis Tools for Power Systems Research and Education," in IEEE Transactions on Power Systems, vol. 26, no. 1, pp. 12-19, Feb. 2011

[39] N. M. M. de Abreu, "Old and new results on algebraic connectivity of graphs," Linear Algebra and its Applications, vol. 423, no. 1, pp. 53-73, May 2007.

[40] B. Mohar, "The Laplacian spectrum of graphs", in Graph Theory, Combinatorics, and Applications, vol. 2, Ed. Y. Alavi, G. Chartrand, O. R. Oellermann, A. J. Schwenk, Wiley, pp. 871-898, 1991.

[41] H. R. Baghaee, M. Mirsalim, G. B. Gharehpetian, and H. A. Talebi "Three phase AC/DC power-flow for balanced/unbalanced microgrids including wind/solar, droop-controlled and electronically-coupled distributed energy resources by RBF neural nets," in IET Power Electronics, vol. 10, no. 3, pp. 313-328, Mar. 2017.

[42] H. R. Baghaee, M. Mirsalim, G. B. Gharehpetian, and H. A. Talebi, "Generalized three phase robust load-flow for radial and meshed power systems with and without uncertainty in energy resources using dynamic radial basis functions neural networks," in Journal of Cleaner Production, vol 174, part C, pp. 96-113, Feb. 2018

Nicolas Retière Nicolas Retière was born in Nantes, France, in 1969. He received the Eng. and Ph.D. degrees from the University of Grenoble Alpes, Grenoble, France, in 1993 and 1997, respectively. He is currently serving as a Professor of electrical engineering with the University of Grenoble Alpes, Grenoble, France, where he holds several responsibility positions and is currently the deputy director of the Physics, Engineering, Materials research departement of the University of Grenoble Alpes . His principal research interests include the modeling, analysis, and design of power systems.

Dinh Truc Ha Truc Dinh Ha was born in Quang Nam, Viet Nam, in 1976. He received the Engineering degree from The University of Da Nang - University of Science and Technology, Viet Nam, in 1999 and Master of Engineering in Electrical power engineering from the University of South Australia, in 2006. He received Ph.D degree. from the University of Grenoble Alpes in 2018. He is now serving as a Lecturer with the Faculty of Electrical Engineering, The University of Da Nang - University of Science and Technology. His research interests include power system analysis and stability.

Jean-Guy Caputo Jean-Guy Caputo was born in Monaco in 1960. He received a master's degree in engineering physics and a master's degree in condensed matter physics in 1983 from the University of Grenoble in France. He obtained a Ph.D. degree in 1986 from the same University. 